\title{
Serum creatinine as a predictor of mortality in patients readmitted to the intensive care unit after cardiac surgery: a retrospective cohort study in China
}

\author{
Jun Zhong ${ }^{1 *}$, Jian Gao ${ }^{2 *}$, Jing-Chao Luo ${ }^{3 \#}$, Ji-Li Zheng ${ }^{1}$, Guo-Wei Tu ${ }^{3}$, Yan Xue ${ }^{1}$ \\ ${ }^{1}$ Department of Nursing, Zhongshan Hospital, Fudan University, Shanghai, China; ${ }^{2}$ Department of Biostatistics, Zhongshan Hospital, Fudan \\ University, Shanghai, China; ${ }^{3}$ Department of Critical Care Medicine, Zhongshan Hospital, Fudan University, Shanghai, China \\ Contributions: (I) Conception and design: J Zhong, J Gao, JC Luo, Y Xue, GW Tu; (II) Administrative support: None; (III) Provision of study \\ materials or patients: J Zhong, Y Xue, JC Luo, GW Tu; (IV) Collection and assembly of data: J Zhong, JC Luo, JL Zheng; (V) Data analysis and \\ interpretation: J Zhong, J Gao, JC Luo, GW Tu; (VI) Manuscript writing: All authors; (VII) Final approval of manuscript: All authors. \\ "These authors contributed equally to this work. \\ Correspondence to: Guo-Wei Tu, MD. Department of Critical Care Medicine, Zhongshan Hospital, Fudan University, Shanghai 200032, China. \\ Email: tu.guowei@zs-hospital.sh.cn; Yan Xue, RN. Department of Nursing, Zhongshan Hospital, Fudan University, Shanghai 200032, China. Email: \\ xue.yan@zs-hospital.sh.cn.
}

Background: Patients readmitted to the intensive care unit (ICU) after cardiac surgery have a high mortality rate. The relationship between renal function and in-hospital mortality in readmitted patients has not been well demonstrated.

Methods: We retrospectively evaluated cardiac surgery patients who were readmitted to the ICU at least once. Data on serum creatinine levels before surgery and on the day of ICU readmission were collected. The estimated glomerular filtration rate (eGFR) was calculated according to the creatinine-based Chronic Kidney Disease-Epidemiology Collaboration equation. We used logistic regression models and restricted cubic spline curves with four knots $(5 \%, 35 \%, 65 \%, 95 \%)$ to investigate the relationship between renal function indicators and mortality.

Results: Of the 184 patients evaluated, 30 patients died during hospitalization, yielding a mortality rate of $16.30 \%$. Cardiac dysfunction $(n=84,45.65 \%)$ and respiration disorder $(n=51,27.72 \%)$ were the most common reasons for ICU readmission. Creatinine [odds ratio (OR): 1.14, $95 \%$ confidence interval (CI): 1.07-1.25] and eGFR (OR: 0.95, 95\% CI: 0.93-0.98) were independently associated with in-hospital mortality after adjusting for various confounders. Both creatinine level and eGFR had a linear association with in-hospital mortality ( $\mathrm{P}$ for non-linearity $>0.05$ ).

Conclusion: Renal function is significantly associated with the in-hospital mortality of patients readmitted to the ICU after cardiac surgery, as evidenced by the independent correlation of both creatinine and eGFR with in-hospital mortality.

Keywords: Readmission, intensive care unit (ICU), cardiac surgery, serum creatinine (SCR), mortality

Submitted Nov 02, 2020. Accepted for publication Feb 06, 2021.

doi: $10.21037 /$ jtd-20-3205

View this article at: http://dx.doi.org/10.21037/jtd-20-3205

\section{Introduction}

Cardiac surgery is a relatively routine procedure in many countries; however, it remains a complex and risky procedure $(1,2)$. Worldwide, nearly 100 million patients undergo cardiac surgery each year, and most require postoperative care in the intensive care unit (ICU) until they stabilize $(3,4)$. However, some patients deteriorate after being transferred to the general ward, eventually 
resulting in unplanned ICU readmission. On average, 7.8\% of patients are readmitted to the ICU after cardiac surgery, and ICU readmission is associated with a higher risk of morbidity, mortality, and incremental health care costs (5-8). However, previous studies mainly focused on the etiology and risk factors for readmission, and the prognostic factors of patients readmitted to the ICU after cardiac surgery remain unclear owing to limited data. Understanding the prognostic factors of in-hospital mortality after ICU readmission would help inform clinicians and improve the treatment outcomes of cardiac surgery in patients who deteriorate postoperatively.

Decreased renal function occurs in patients undergoing both cardiac and non-cardiac surgeries and has been reported to be associated with high mortality (9-14). The purpose of this study was to investigate whether the renal function of patients readmitted to the ICU after cardiac surgery was associated with in-hospital mortality.

We present the following article in accordance with the STROBE reporting checklist (available at http://dx.doi. org/10.21037/jtd-20-3205).

\section{Methods}

\section{Study design and patients}

This was a retrospective cohort study of patients who underwent cardiac surgery between January 1, 2018, and December 31, 2019, in Zhongshan Hospital, Shanghai, China and were readmitted to the ICU at least once during their hospitalization. We defined ICU readmission as the second or subsequent ICU admission of a patient due to deterioration of clinical status and vital signs that warranted advanced monitoring and therapy. Patients younger than 18 years were excluded.

The patients received standard anesthesia and cardiac surgical procedures and were thereafter directly admitted to the ICU for postoperative management. A team of intensivists, nurses, and respiratory therapists managed the patients during their ICU stay. The ICU nurse-patient ratio is $1: 2$ during the daytime and $1: 3$ at night time. Intensivists decided to transfer patients out of the ICU based on the patients' general conditions and laboratory results. Meanwhile, the cardiac surgeon made decisions about the need for ICU readmission from the general ward. For patients who were readmitted to the ICU more than once during a single hospitalization, the data of the last readmission were included in the analysis.
The study was conducted in accordance with the Declaration of Helsinki (as revised in 2013). The study was approved by the Ethical Committee of Zhongshan hospital, Fudan University (B2019-075R), and individual consent for this retrospective analysis was waived.

\section{Data collection}

We obtained all data from the patients' electronic medical record system. Preoperative characteristics included age, sex, body mass index (BMI), New York Heart Association (NYHA) functional classification, hypertension, diabetes, chronic kidney disease (CKD), history of cardiac surgery, and European System for Cardiac Operative Risk Evaluation (Euro score). The cardiac surgeon assessed the NYHA functional classification. Postoperative data included the type of surgery, Acute Physiology and Chronic Health Evaluation II (APACHE II) scores, left ventricular ejection fraction (LVEF), the main cause of ICU readmission, aspartate aminotransferase (AST), alanine aminotransferase (ALT), lactic acid, and length of ICU and hospital stay. LVEF was obtained from transthoracic echocardiography. The main cause for ICU readmission was determined based on the medical records.

We collected data on the serum creatinine (SCR) levels before surgery and on the day of ICU readmission, and we calculated the estimated glomerular filtration rate (eGFR) according to the creatinine-based Chronic Kidney DiseaseEpidemiology Collaboration (CKD-EPI) equation. For women, eGFR was calculated as $144 \times(\mathrm{SCR} / 62)-\mathrm{k} \times$ $0.993^{\text {age }}(\mathrm{k}=0.329$, if SCR was $\leq 62 \mu \mathrm{mol} / \mathrm{L} ; \mathrm{k}=1.209$, if SCR $>62 \mu \mathrm{mol} / \mathrm{L})$. For men, it was calculated as $144 \times(\mathrm{SCR} / 80)$ $-\mathrm{k} \times 0.993^{\mathrm{age}}(\mathrm{k}=0.411$ if SCR was $\leq 80 \mu \mathrm{mol} / \mathrm{L} ; \mathrm{k}=1.209$ if SCR $>80 \mu \mathrm{mol} / \mathrm{L})$. We also calculated the $\Delta \mathrm{SCR}$ and $\triangle \mathrm{eGFR}$ as variations of indicators before surgery and on the day of ICU readmission.

\section{Statistical analysis}

The primary study endpoint was all-cause in-hospital mortality. Continuous variables were expressed as the mean and standard deviation (SD) or median and interquartile range (IQR), depending on the nature and distribution of the variables. Categorical variables were expressed as frequency counts with percentages. Continuous variables were compared using the Wilcoxon rank-sum test or Student's independent $t$-test, as appropriate, whereas categorical variables were compared using the chi-square 
test or Fisher's exact test. Logistic regression models were used to investigate the relationship between renal function indicators and mortality in univariable and multivariable analyses. We established unadjusted, moderately adjusted, and fully adjusted models. In the moderately adjusted models, SCR, eGFR, $\triangle \mathrm{SCR}$, and $\triangle \mathrm{eGFR}$ at the time of ICU readmission were individually entered and adjusted for lactic acid, and infection. In the fully adjusted models, they were adjusted for age, sex, BMI, NYHA functional classification, Euro score, APACHE II scores, aortic surgery, AST, ALT, lactic acid, and infection.

Restricted cubic spline curves with four knots (5\%, 35\%, $65 \%, 95 \%)$ were used to determine the association between creatinine and death. We further applied a two-piecewise multivariable logistic regression model to examine the threshold effect of creatinine on death according to the smooth curve fitting. Using a tail and error, we determined the threshold level (i.e., turning point) of creatinine at which the relationship between creatinine and death began to change and became notable. The trial inflection point was moved along a pre-defined interval and detected the inflection point that gave the maximum model likelihood. This method was also used for eGFR, $\triangle \mathrm{SCR}$, and $\triangle \mathrm{eGFR}$. We also tested for potential non-linearity by using a likelihood ratio test, comparing the model with only a linear term against the model with linear and cubic spline terms. Results of the logistic regression model were presented as odds ratios (ORs) and 95\% confidence intervals (CIs). All statistical analyses were performed using SPSS version 22.0 (IBM, New York, NY, USA) and R statistical software (R, version 3.5.1; $R$ Project). Statistical significance was defined as $\mathrm{P}<0.05$.

\section{Results}

\section{Patient characteristics}

Among the 9,747 consecutive adult patients (age $\geq 18$ years) who underwent cardiac surgery, 184 patients $(1.89 \%)$ were readmitted to the ICU at least once and were evaluated. Of the 184 patients, 30 patients $(16.30 \%)$ died during hospitalization. The patient characteristics according to survival status are shown in Table 1. There were no significant differences in age, sex, BMI, LVEF, hypertension, diabetes, CKD, history of cardiac surgery, SCR, and eGFR between the two groups (all $\mathrm{P}>0.05$ ). However, an increased hazard of death was observed in patients who underwent aortic surgery and who had higher Euro and APACHE II scores and elevated levels of AST, ALT, and lactic acid. SCR, eGFR, $\triangle \mathrm{SCR}$, and $\triangle \mathrm{eGFR}$ at the time of ICU readmission were also significantly associated with in-hospital mortality (Table 1).

\section{Main causes of readmission}

Cardiac dysfunction $(\mathrm{n}=84,45.65 \%)$ and respiration disorder $(\mathrm{n}=51,27.72 \%)$ were the most common reasons for ICU readmission. The other reasons were neurological problems, acute renal failure, infection, cardiac tamponade, or bleeding. The causes of readmission are shown in Table 2.

\section{Association between renal function and in-bospital mortality}

The multivariable regression analysis indicated that SCR, eGFR, $\triangle \mathrm{SCR}$, and $\triangle \mathrm{eGFR}$ at the time of readmission were all significantly associated with in-hospital mortality (Table 3). The crude model showed a strong correlation between all the renal function indicators (SCR, eGFR, $\triangle \mathrm{SCR}$, and $\triangle \mathrm{eGFR}$ ) and in-hospital mortality. In the moderately adjusted model, a significant association was evident after separate adjustments for lactic acid and infection (other adjusted indicators and overall effect value see Table S1). The significant association remained after adjustments for selected clinical predictors of survival (age, sex, BMI, NYHA functional classification, Euro score, APACHE II score, aortic surgery, AST, ALT, lactic acid, and infection) in the fully adjusted model (result of Poisson regression see Table S2).

Four smooth curves were fitted, and the relationship between SCR, eGFR, $\triangle \mathrm{SCR}$, and $\triangle \mathrm{eGFR}$ with in-hospital mortality was assessed. The result showed that SCR, eGFR, $\triangle \mathrm{SCR}$, and $\triangle \mathrm{eGFR}$ were linearly related to in-hospital mortality after comparing the model with the non-linear predictor and linear predictor by likelihood ratio test $(\mathrm{P}$ for linearity $<0.05$, P for non-linearity $>0.05$ ) (Figure 1 ).

\section{Discussion}

Decreased renal function is associated with a high mortality rate and occurs in patients undergoing cardiac surgery, but its prognostic impact in patients readmitted to the ICU after cardiac surgery has not yet been clarified. The present study found a $16.3 \%$ ( $\mathrm{n}=30$ deaths) mortality rate in 184 patients who were readmitted to the ICU after cardiac surgery. Importantly, decreased renal function was strongly 
Table 1 Patient characteristics by survival status $(\mathrm{n}=184)$

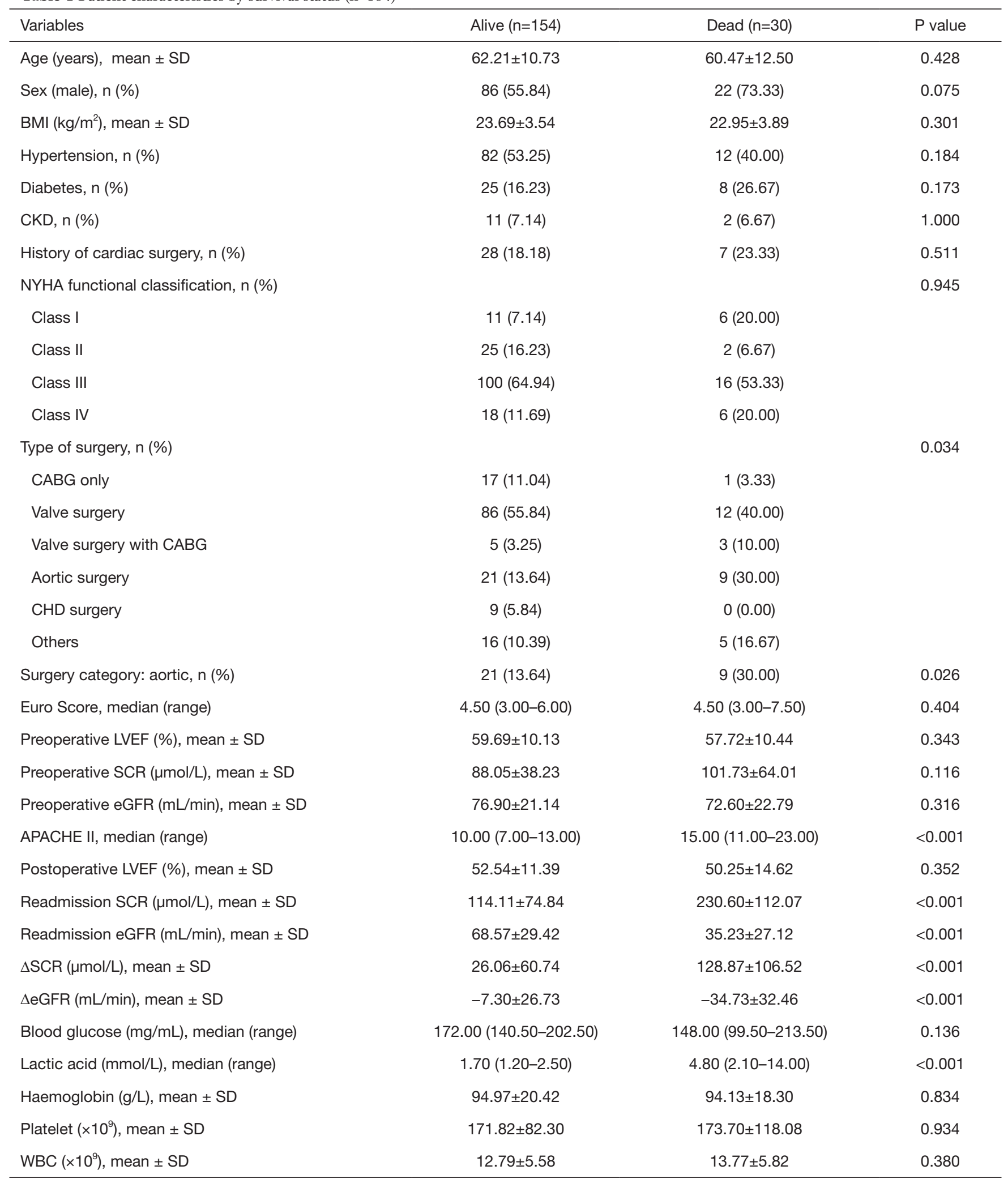

Table 1 (continued) 
Table 1 (continued)

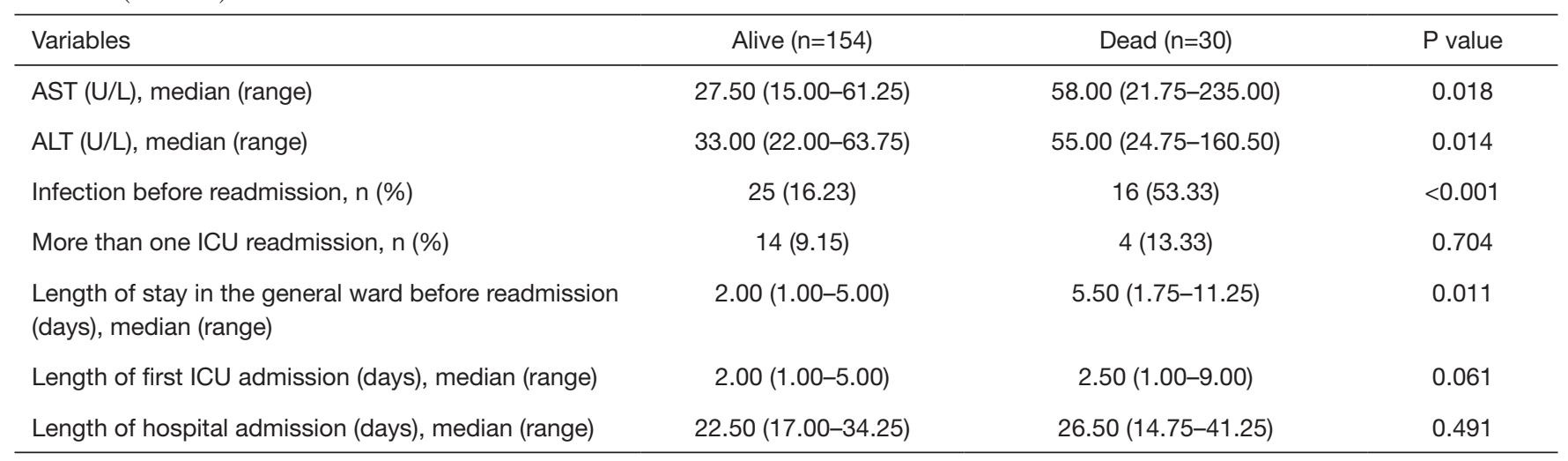

$\mathrm{SD}$, standard deviation; BMI, body mass index; CKD, chronic kidney disease; NYHA, New York Heart Association; CABG, coronary artery bypass graft surgery; CHD, congenital heart disease; LVEF, left ventricular ejection fraction; SCR, serum creatinine; eGFR, estimated glomerular filtration rate; WBC, white blood cell; AST, aspartate aminotransferase; ALT, alanine aminotransferase; ICU, intensive care unit.

Table 2 Main causes of ICU readmission

\begin{tabular}{lccc}
\hline Main causes of readmission & All patients $(\mathrm{n}=184)$ & Alive $(\mathrm{n}=154)$ & Dead $(\mathrm{n}=30)$ \\
\hline Cardiac dysfunction, $\mathrm{n}(\%)$ & $84(45.65)$ & $72(46.75)$ & $12(40.00)$ \\
Respiratory disorder, $\mathrm{n}(\%)$ & $51(27.72)$ & $49(31.82)$ & $2(6.67)$ \\
Neurological problems, $\mathrm{n}(\%)$ & $22(11.96)$ & $17(11.04)$ & $5(16.67)$ \\
Acute renal failure, $\mathrm{n}(\%)$ & $13(7.07)$ & $6(3.90)$ & $7(23.33)$ \\
Infection, $\mathrm{n}(\%)$ & $6(3.26)$ & $4(2.60)$ & $2(6.67)$ \\
Cardiac tamponade/bleeding, $\mathrm{n}(\%)$ & $8(4.35)$ & $6(3.90)$ & $2(6.67)$ \\
\hline
\end{tabular}

ICU, intensive care unit.

associated with mortality among these patients.

A retrospective analysis of 10,992 consecutive adult cardiac surgery patients found a $23.9 \%$ in-hospital mortality rate for patients readmitted to the ICU for any cause (15). A multi-center study also reported a 5-fold higher rate of in-hospital mortality in patients readmitted to the ICU as compared to those who were not readmitted $(20.7 \%$ vs. $4.4 \%$ ) (16). In another retrospective observational cohort study, the in-hospital mortality rate was $6.5 \%$ in readmitted patients, and the late mortality rate was significantly higher in the readmission group than that of the nonreadmission group $(22.2 \%$ vs. $3.2 \%, \mathrm{P}<0.001)(17)$. The different mortality rates reported in these studies may be related to the different standards of ICU readmission and the proportion of complex operations. In the present study, the primary causes of ICU readmission were respiratory disorders and cardiac dysfunction, consistent with the findings of many previous studies $(7,17-19)$. Respiratory and circulatory failure are common complications after cardiac surgery. The changes in vital signs are also the first and most observable signs in patients with deterioration.

The etiology and risk factors for ICU readmission are well described in the literature. However, the determinants of patient mortality after readmission have not been described due to the limitations of the sample size. In this study, decreased renal function was strongly associated with in-hospital death among patients who were readmitted to the ICU. The risk of death increased by 0.14 -fold for every $10 \mathrm{mmol} / \mathrm{L}$ increase in SCR. Similar results were obtained after calculating the eGFR using the creatinine-based CKD-EPI equation. SCR is important for the assessment of renal function and the prediction of the risk of mortality after cardiac surgery. Studies in the last 20 years suggested that decreased renal function was associated with poor prognosis in both cardiac and noncardiac surgery patients (20-22). However, these studies did not separately evaluate 
Table 3 Multivariable regression analysis for the effect of creatinine and eGFR on survival

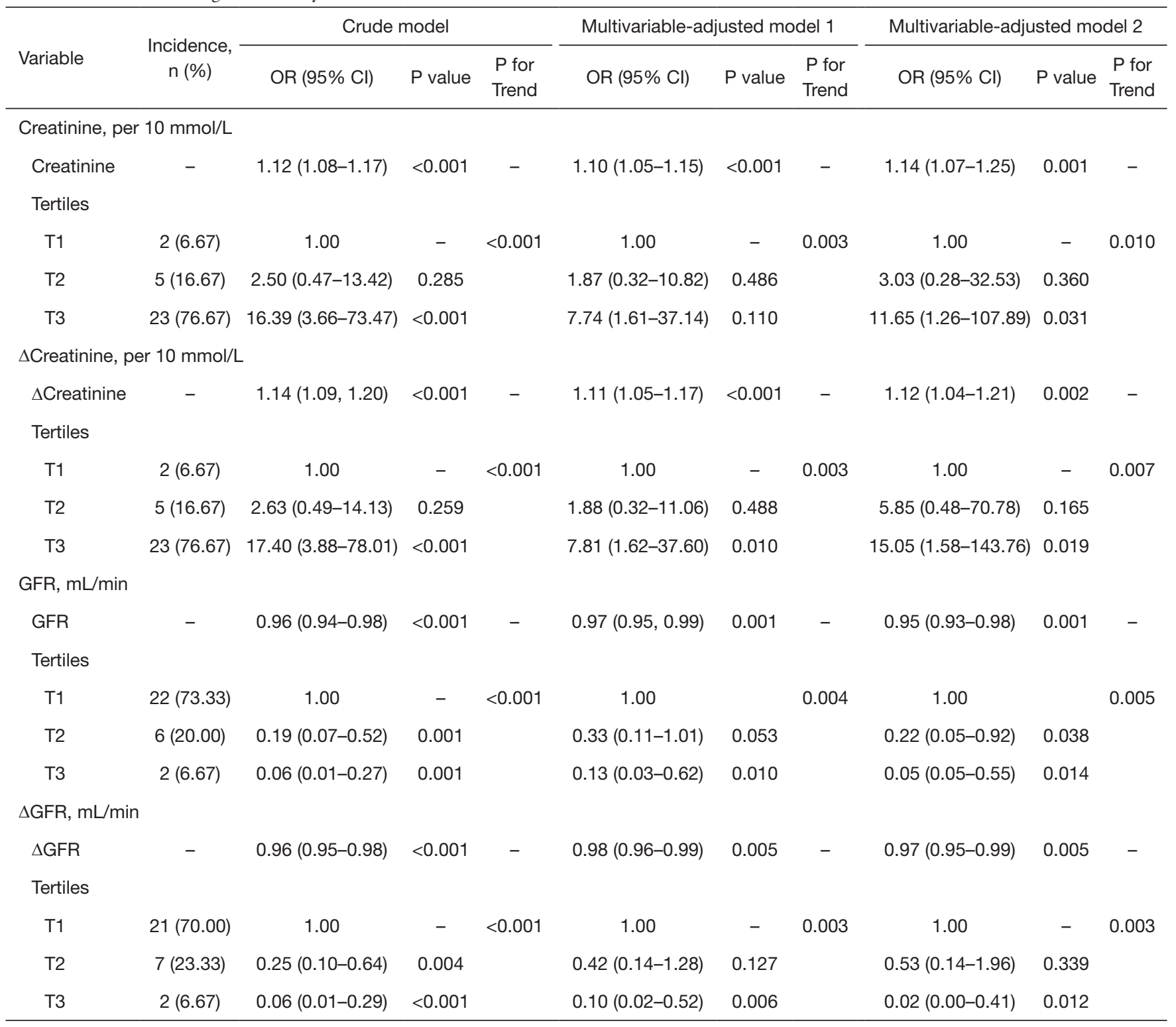

Model 1: adjusted for lactic acid and infection; Model 2: adjusted for age, sex, BMI, NYHA functional classification, aortic surgery, Euro score, APACHE II, AST, ALT, lactic acid, and infection. eGFR, estimated glomerular filtration rate; OR, odds ratio; Cl, confidence interval; GFR, glomerular filtration rate; BMI, body mass index; NYHA, New York Heart Association; APACHE II, Acute Physiology and Chronic Health Evaluation II; AST, aspartate aminotransferase; ALT, alanine aminotransferase.

the patients who were readmitted to the ICU.

Through the analysis of $\triangle \mathrm{SCR}$ and $\triangle \mathrm{eGFR}$, we found that the variation in SCR and eGFR is also strongly associated with in-hospital mortality regardless of the level of preoperative renal function. No patient with an $\triangle \mathrm{eGFR}$ $>0$ died. These findings further suggest that secondary renal injury after cardiac surgery is an important risk factor for death in ICU re-admission patients.
There are several possible explanations for the results of the study. First, more than $70 \%$ of the patients in our study were readmitted to the ICU due to cardiopulmonary insufficiency, which may result in a series of physiological changes. Acute kidney injury (AKI) developed after cardiac surgery was also labeled as acute cardiorenal syndrome type I (CRS-I) (23). The occurrence of CRS-I in cardiac surgical patients often indicated the worsening of cardiac 

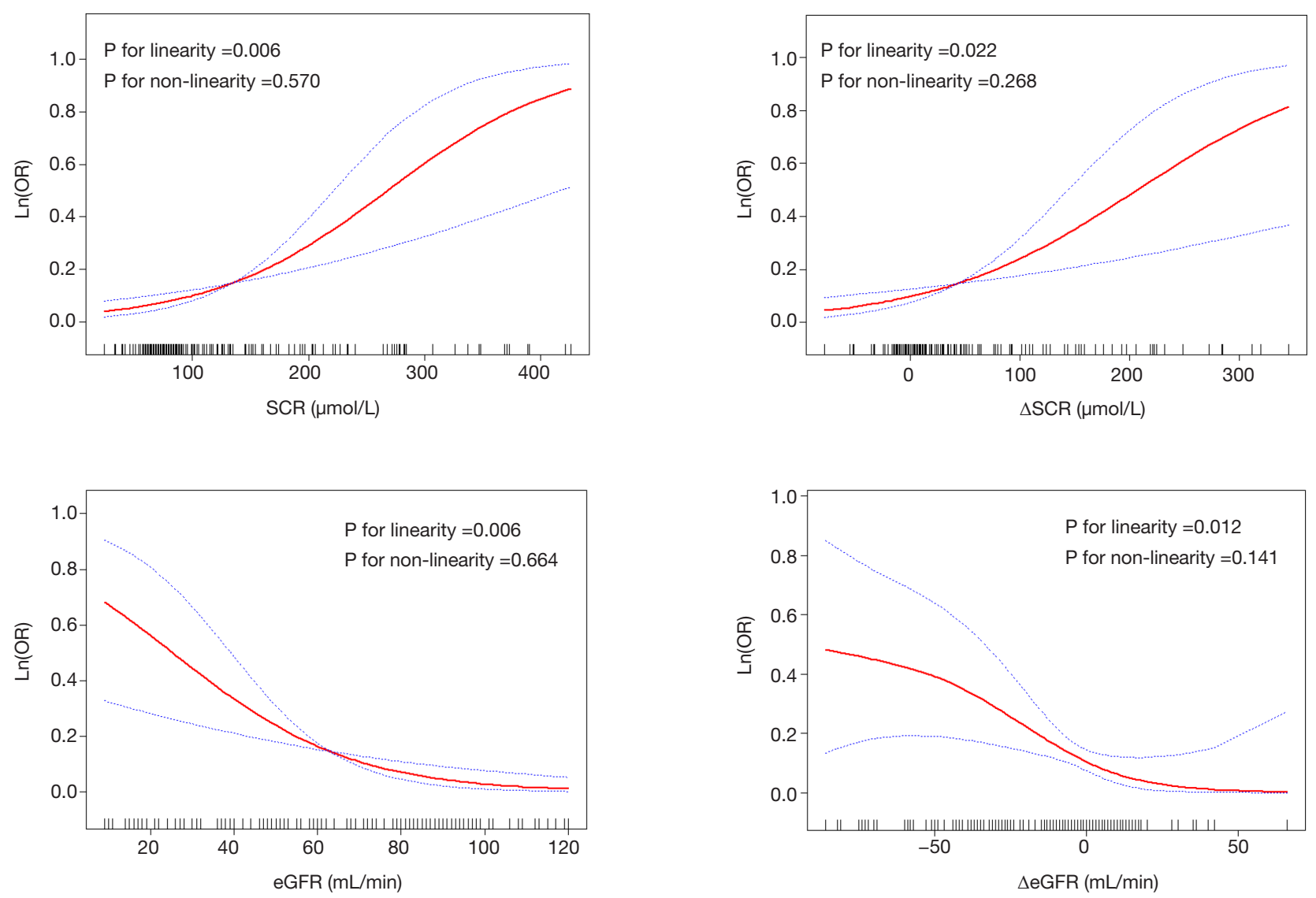

Figure 1 Relationship between renal function indicators and $\log$ odds ratio (OR) of death after controlling for potential confounding variables (multivariable OR, 95\% confidence intervals, and P value are shown). SCR, serum creatinine; eGFR, estimated glomerular filtration rate.

function. Second, previous studies have demonstrated that patients with CRS-I after cardiac surgery had a higher risk of mortality (24). Increased level of SCR could be the surrogate marker of many fatal complications such as perioperative blood loss, acute coronary syndrome, acute heart failure, prolonged period on cardiopulmonary bypass, or postoperative infections. All these events can result in additional deterioration of kidney function, and some of these are strongly associated with postoperative mortality. Third, AKI can further worsen cardiac function and form a vicious circle. The fluid overload caused by the decrease of renal function will increase the volume of circulating blood and make venous and pulmonary circulation congestive, which further aggravates the symptoms of congestive heart failure in these patients (25). In summary, an increased level of SCR represents a more severe cardiac decompensation in patients readmitted to the ICU after cardiac surgery, and thus predicts in-hospital mortality.

\section{Limitation}

The present study is a single-center retrospective study based on consecutive data from a large tertiary cardiac surgery center in China. There were very few missing data and cases excluded from the analysis. However, some limitations of the study should be addressed. First, we controlled potential confounders to the best of our ability, yet residual confounding by other unmeasured or unknown factors might still exist. Second, the small sample size of the death event may result in overfitting. This would bias results toward the null and result in an underestimation of the association between creatinine and death. The readmission to the ICU was a rare event. To our limited knowledge, this study has the largest sample size of cardiac surgery patients readmitted to the ICU in China. Third, AKI classification was not included in the analysis because patients were not routinely assessed for AKI after returning to the general 
ward. Furthermore, the conclusion was made based on a single ICU study. Multi-center studies are needed to verify our findings.

\section{Conclusions}

This single-center study found that $1.89 \%$ of patients who underwent cardiac surgery were re-admitted to the ICU, and $16.30 \%$ of them died in the hospital. Cardiac dysfunction and respiration disorder were the most common reasons for readmission. Both SCR and eGFR were significantly associated with in-hospital mortality among readmitted patients, highlighting the predictive value of SCR for mortality after cardiac surgery. The intensivists should pay more attention to renal function in ICU re-admitted cardiac surgical patients.

\section{Acknowledgments}

The authors thank the nurses at the cardiac surgery intensive care unit in Zhongshan Hospital, Fudan University, for the help of data collection. Also, the authors would like to thank Editage (www.editage.cn) for English language editing.

Funding: This article was supported by grants from the Fudan-Fosun Nursing Research Fund (FNF201816), Shanghai Municipal Health Commission (2019ZB0105), Program of Shanghai Academic/Technology Research Leader (20XD1421000), Natural Science Foundation of Shanghai (20ZR1411100), National Natural Science Foundation of China (82070085), Clinical Research Funds of Zhongshan Hospital (2020ZSLC38 and 2020ZSLC27), and Smart Medical Care of Zhongshan Hospital (2020ZHZS01).

\section{Footnote}

Reporting Checklist: The authors have completed the STROBE reporting checklist. Available at http://dx.doi. org/10.21037/jtd-20-3205

Data Sharing Statement: Available at http://dx.doi. org/10.21037/jtd-20-3205

Conflicts of Interest: All authors have completed the ICMJE uniform disclosure form (available at http://dx.doi. org/10.21037/jtd-20-3205). The authors have no conflicts of interest to declare.
Ethical Statement: The authors are accountable for all aspects of the work in ensuring that questions related to the accuracy or integrity of any part of the work are appropriately investigated and resolved. The study was conducted in accordance with the Declaration of Helsinki (as revised in 2013). The study was approved by the Ethical Committee of Zhongshan hospital, Fudan University (B2019-075R), and individual consent for this retrospective analysis was waived.

Open Access Statement: This is an Open Access article distributed in accordance with the Creative Commons Attribution-NonCommercial-NoDerivs 4.0 International License (CC BY-NC-ND 4.0), which permits the noncommercial replication and distribution of the article with the strict proviso that no changes or edits are made and the original work is properly cited (including links to both the formal publication through the relevant DOI and the license). See: https://creativecommons.org/licenses/by-nc-nd/4.0/.

\section{References}

1. Curiel-Balsera E, Mora-Ordonez JM, Castillo-Lorente $\mathrm{E}$, et al. Mortality and complications in elderly patients undergoing cardiac surgery. J Crit Care 2013;28:397-404.

2. Funkat AK, Beckmann A, Lewandowski J, et al. Cardiac surgery in Germany during 2011: a report on behalf of the German Society for Thoracic and Cardiovascular Surgery. Thorac Cardiovasc Surg 2012;60:371-82.

3. Smilowitz NR, Berger JS. Perioperative Management to Reduce Cardiovascular Events. Circulation 2016;133:1125-30.

4. Weiser TG, Haynes AB, Molina G, et al. Estimate of the global volume of surgery in 2012: an assessment supporting improved health outcomes. Lancet 2015;385 Suppl 2:S11.

5. Bardell T, Legare JF, Buth KJ, et al. ICU readmission after cardiac surgery. Eur J Cardiothorac Surg 2003;23:354-9.

6. Benetis R, Sirvinskas E, Kumpaitiene B, et al. A casecontrol study of readmission to the intensive care unit after cardiac surgery. Med Sci Monit 2013;19:148-52.

7. Joskowiak D, Wilbring M, Szlapka M, et al. Readmission to the intensive care unit after cardiac surgery: a singlecenter experience with 7105 patients. J Cardiovasc Surg (Torino) 2012;53:671-6.

8. Thomson R, Fletcher N, Valencia O, et al. Readmission to the Intensive Care Unit Following Cardiac Surgery: A Derived and Validated Risk Prediction Model in 4,869 
Patients. J Cardiothorac Vasc Anesth 2018;32:2685-91.

9. Amini S, Najafi MN, Karrari SP, et al. Risk Factors and Outcome of Acute Kidney Injury after Isolated CABG Surgery: a Prospective Cohort Study. Braz J Cardiovasc Surg 2019;34:70-5.

10. Ishani A, Nelson D, Clothier B, et al. The magnitude of acute serum creatinine increase after cardiac surgery and the risk of chronic kidney disease, progression of kidney disease, and death. Arch Intern Med 2011;171:226-33.

11. Li S, Wang S, Priyanka P, et al. Acute Kidney Injury in Critically Ill Patients After Noncardiac Major Surgery: Early Versus Late Onset. Crit Care Med 2019;47:e437-44.

12. Pickering JW, Blunt IRH, Than MP. Acute Kidney Injury and mortality prognosis in Acute Coronary Syndrome patients: A meta-analysis. Nephrology (Carlton) 2018;23:237-46.

13. Pourafkari L, Arora P, Porhomayon J, et al. Acute kidney injury after non-cardiovascular surgery: risk factors and impact on development of chronic kidney disease and longterm mortality. Curr Med Res Opin 2018;34:1829-37.

14. Shen W, Aguilar R, Montero AR, et al. Acute Kidney Injury and In-Hospital Mortality after Coronary Artery Bypass Graft versus Percutaneous Coronary Intervention: A Nationwide Study. Am J Nephrol 2017;45:217-25.

15. Litwinowicz R, Bartus K, Drwila R, et al. In-hospital mortality in cardiac surgery patients after readmission to the intensive care unit: a single-center experience with 10,992 patients. J Cardiothorac Vasc Anesth 2015;29:570-5.

16. Renton J, Pilcher DV, Santamaria JD, et al. Factors associated with increased risk of readmission to intensive care in Australia. Intensive Care Med 2011;37:1800-8.

17. Kang YA. Risk Factors and Outcomes Associated With

Cite this article as: Zhong J, Gao J, Luo JC, Zheng JL, Tu GW, Xue Y. Serum creatinine as a predictor of mortality in patients readmitted to the intensive care unit after cardiac surgery: a retrospective cohort study in China. J Thorac Dis 2021;13(3):1728-1736. doi: 10.21037/jtd-20-3205
Readmission to the Intensive Care Unit After Cardiac Surgery. AACN Adv Crit Care 2016;27:29-39.

18. Giakoumidakis K, Eltheni R, Patelarou A, et al. Incidence and predictors of readmission to the cardiac surgery intensive care unit: A retrospective cohort study in Greece. Ann Thorac Med 2014;9:8-13.

19. Litmathe J, Kurt M, Feindt P, et al. Predictors and outcome of ICU readmission after cardiac surgery. Thorac Cardiovasc Surg 2009;57:391-4.

20. Brown JR, Kramer RS, Coca SG, et al. Duration of acute kidney injury impacts long-term survival after cardiac surgery. Ann Thorac Surg 2010;90:1142-8.

21. Lassnigg A, Schmidlin D, Mouhieddine M, et al. Minimal changes of serum creatinine predict prognosis in patients after cardiothoracic surgery: a prospective cohort study. J Am Soc Nephrol 2004;15:1597-605.

22. Mooney JF, Croal BL, Cassidy S, et al. Relative value of cystatin $\mathrm{C}$ and creatinine-based estimates of glomerular filtration rate in predicting long-term mortality after cardiac surgery: a cohort study. BMJ Open 2019;9:e029379.

23. Ronco C, Cicoira M, McCullough PA. Cardiorenal syndrome type 1: pathophysiological crosstalk leading to combined heart and kidney dysfunction in the setting of acutely decompensated heart failure. J Am Coll Cardiol 2012;60:1031-42.

24. Vandenberghe W, Gevaert S, Kellum JA, et al. Acute Kidney Injury in Cardiorenal Syndrome Type 1 Patients: A Systematic Review and Meta-Analysis. Cardiorenal Med 2016;6:116-28.

25. Schefold JC, Filippatos G, Hasenfuss G, et al. Heart failure and kidney dysfunction: epidemiology, mechanisms and management. Nat Rev Nephrol 2016;12:610-23. 
Supplementary

Table S1 OR and 95\% CI of different variables

\begin{tabular}{|c|c|c|c|c|c|c|c|c|}
\hline \multirow{2}{*}{ Variables } & \multicolumn{2}{|c|}{ SCR (per 10 mmol/L) } & \multicolumn{2}{|c|}{$\Delta \mathrm{SCR}$ (per $10 \mathrm{mmol} / \mathrm{L})$} & \multicolumn{2}{|l|}{ eGFR } & \multicolumn{2}{|l|}{$\Delta \mathrm{eGFR}$} \\
\hline & OR $(95 \% \mathrm{Cl})$ & $P$ value & OR $(95 \% \mathrm{Cl})$ & $P$ value & OR $(95 \% \mathrm{Cl})$ & $P$ value & OR $(95 \% \mathrm{Cl})$ & $P$ value \\
\hline Crude & $1.122(1.076,1.170)$ & $<0.001$ & $1.142(1.087,1.200)$ & $<0.001$ & $0.960(0.944,0.976)$ & $<0.001$ & $0.966(0.952,0.981)$ & $<0.001$ \\
\hline \multicolumn{9}{|c|}{ Included one covariate in a separate logistic regression model } \\
\hline+ Age & $1.130(1.081-1.180)$ & $<0.001$ & $1.147(1.091-1.206)$ & $<0.001$ & $0.956(0.939-0.973)$ & $<0.001$ & $0.966(0.952-0.981)$ & $<0.001$ \\
\hline+ Sex & $1.124(1.077-1.173)$ & $<0.001$ & $1.146(1.089-1.206)$ & $<0.001$ & $0.958(0.942-0.975)$ & $<0.001$ & $0.966(0.952-0.981)$ & $<0.001$ \\
\hline$+\mathrm{BMl}$ & $1.128(1.080-1.178)$ & $<0.001$ & $1.144(1.088-1.203)$ & $<0.001$ & $0.959(0.943-0.976)$ & $<0.001$ & $0.966(0.952-0.981)$ & $<0.001$ \\
\hline+ NYHA functional classification & $1.128(1.080-1.178)$ & $<0.001$ & $1.148(1.091-1.208)$ & $<0.001$ & $0.958(0.942-0.975)$ & $<0.001$ & $0.965(0.950-0.980)$ & $<0.001$ \\
\hline+ Aortic surgery & $1.123(1.076-1.173)$ & $<0.001$ & $1.142(1.086-1.200)$ & $<0.001$ & $0.959(0.943-0.976)$ & $<0.001$ & $0.964(0.950-0.979)$ & $<0.001$ \\
\hline +Euro score & $1.120(1.073-1.169)$ & $<0.001$ & 1.139 (1.084-1.198) & $<0.001$ & $0.961(0.945-0.978)$ & $<0.001$ & $0.966(0.952-0.981)$ & $<0.001$ \\
\hline +APACHE II & $1.111(1.061-1.164)$ & $<0.001$ & $1.128(1.069-1.190)$ & $<0.001$ & $0.963(0.944-0.982)$ & $<0.001$ & $0.971(0.955-0.987)$ & 0.001 \\
\hline+ AST & $1.121(1.075-1.169)$ & $<0.001$ & $1.140(1.085-1.198)$ & $<0.001$ & $0.961(0.944-0.977)$ & $<0.001$ & $0.967(0.952-0.982)$ & $<0.001$ \\
\hline$+\mathrm{ALT}$ & $1.120(1.074-1.168)$ & $<0.001$ & $1.139(1.084-1.197)$ & $<0.001$ & $0.961(0.945-0.977)$ & $<0.001$ & $0.967(0.953-0.982)$ & $<0.001$ \\
\hline +Lactic acid & $1.115(1.067-1.166)$ & $<0.001$ & $1.126(1.069-1.185)$ & $<0.001$ & $0.964(0.947-0.982)$ & $<0.001$ & $0.974(0.959-0.990)$ & 0.001 \\
\hline +Infection & $1.107(1.060-1.157)$ & $<0.001$ & $1.125(1.068-1.185)$ & $<0.001$ & $0.965(0.949-0.982)$ & $<0.001$ & $0.972(0.957-0.987)$ & $<0.001$ \\
\hline \multicolumn{9}{|c|}{ Included two covariates in a separate logistic regression model } \\
\hline +Lactic acid + APACHE II & $1.113(1.059-1.169)$ & $<0.001$ & $1.118(1.057-1.182)$ & $<0.001$ & $0.964(0.945-0.985)$ & 0.001 & $0.978(0.961-0.995)$ & 0.011 \\
\hline +APACHE II + infection & $1.102(1.051-1.156)$ & $<0.001$ & $1.116(1.055-1.180)$ & $<0.001$ & $0.965(0.946-0.984)$ & $<0.001$ & $0.974(0.958-0.991)$ & 0.002 \\
\hline +Lactic acid + infection & 1.100 (1.049-1.153) & $<0.001$ & 1.107 (1.047-1.171) & $<0.001$ & $0.970(0.952-0.988)$ & 0.001 & $0.978(0.962-0.993)$ & 0.005 \\
\hline
\end{tabular}

OR, odds ratio; $\mathrm{Cl}$, confidence interval; SCR, serum creatinine; eGFR, estimated glomerular filtration rate; APACHE II, Acute Physiology and Chronic Health Evaluation II; AST, aspartate aminotransferase; ALT, alanine aminotransferase. 
Table S2 Relationship between creatinine and death by Poisson regression and logistic regression

\begin{tabular}{|c|c|c|c|c|}
\hline Variables & \multicolumn{2}{|c|}{ Logistic regression } & \multicolumn{2}{|c|}{ Poisson regression } \\
\hline Creatinine, per $10 \mathrm{mmol} / \mathrm{L}$ & $1.14(1.07-1.25)$ & 0.001 & $1.09(1.06-1.11)$ & $<0.001$ \\
\hline$\Delta$ Creatinine, per $10 \mathrm{mmol} / \mathrm{L}$ & $1.12(1.04-1.21)$ & 0.002 & $1.09(1.07-1.11)$ & $<0.001$ \\
\hline $\mathrm{GFR}, \mathrm{mL} / \mathrm{min}$ & $0.95(0.93-0.98)$ & 0.001 & $0.96(0.95-0.98)$ & $<0.001$ \\
\hline
\end{tabular}

Adjusted for age, sex, BMI, NYHA functional classification, aortic surgery, Euro score, APACHE II, AST, ALT, lactic acid, and infection. OR, odds ratio; Cl, confidence interval; IRR, incidence rate ratio; GFR, glomerular filtration rate; BMI, body mass index; NYHA, New York Heart Association; APACHE II, Acute Physiology and Chronic Health Evaluation II; AST, aspartate aminotransferase; ALT, alanine aminotransferase. 